\title{
Extraction of Lignin from Hemp Shives
}

\author{
Martins Andzs, Janis Gravitis, Andris Veveris \\ 1 - Wood Materials and Technology, Department of Wood Processing, Latvia University of Agriculture, \\ Dobeles str. 41, Jelgava, LV-3001, tel.: +371 29397439 \\ 2 - Head of laboratory, Latvian State Institute of Wood Chemistry, Dzerbenes 27, Riga, LV 1006, Latvia, tel.: \\ +371 67553137, fax: +37167550635 \\ 3 - Latvian State Institute of Wood Chemistry. Dzerbenes 27, Riga, LV 1006, Latvia, tel.: +371 67553137, \\ fax: +37167550635
}

\begin{abstract}
Industrial hemp contains 65-80\% shives of the stalk and are composed of libriform fibres who are high lignin content. Steam explosion (SE) auto-hydrolysis is a simple treatment of biomass (agricultural waste, logging residues, etc.) by saturated only steam without any additional reagents, usually at pressures 32 atmospheres. This is the basic method how to produce lignin without any dash. Analogy if there is the most sever SE and more are destroyed hemp shives, the more lignin can extract. The aim is to definite the bulk volume, content of lignin in 'Belobrzesky' hemp variety depending on the SE treatment factor, doing extraction with $\mathrm{H}^{2} \mathrm{O}$ and $\mathrm{NaOH} 0.4 \%$ and compare without going content of hemp shives lignin.
\end{abstract}

Keywords - hemp shives, steam explosion, lignin, extraction.

\section{INTRODUCTION}

All over the world there are not many substances that chemical structure is not know. We know the structure of cellulose other polysacharides, albumen. The chemical content of lignin is not fixed because it is highly variable structure, any extraction method of lignin of plants and wood, it gives deep changes in structure because the essential meaning of distribution method. The size Steam explosion (SE) fibre depends on the SE way of processing. If the way of processing is longer, it gives more smaller fibre particles [Zakis, 2008].

The extraction with water and alkaline off the mass off SE gives essential influence on the size and elasticity because hemicellulose has been washed out.

Extraction of lignes is made in two stages: dissolving the holocellulose in water leaving the insoluble cellulose and lignin in the residue further processed in alkali to dissolve lignin.

Lignin is one of the most abundant natural raw materials available on earth, second to cellulose by mass and even first by solar energy. Presently only about $2 \%$ of lignin contained in lignosulphonates from sulphite pulping [Gargulak and Lebo, 2000] is recovered in the craft process.

Lignin (Fig. 1) can be used as binder to plywood, fibreboards and particle boards. Lignin is also used as a component in coatings and foams. Lignin and its modification serve as dispersants, slow-release fertilizers, sorbents, carbon fibres, insecticides, and plant hormones. Commercial potential of many lignin derivatives showing good properties is still unclear. Lignin obtained by conventional technologies containing sulphur is often burned and used as a heat source. The SE lignin is sulphur-free and chemically reactive. During the SE treatment lignin coalescing in spheres of diameter to some micrometers on the surface of cellulose fibres promotes enzymatic attack on cellulose [Gravitis et al., 2010]. The aim of the study is to research extraction lignin from hemp shives, and study the effects of SE on the outcome of the lignin.

II MATERIALS AND METHODS

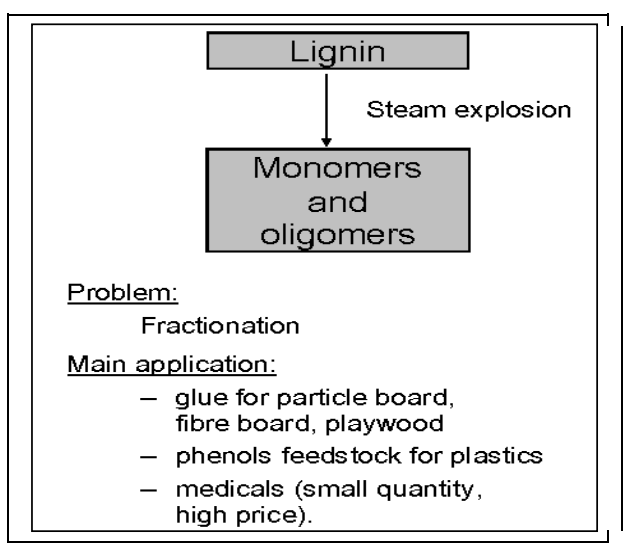

Fig. 1. Application of lignin from SE pulp [Gravitis, 1996].

The desintegration of hemp shives with steam explosion with the method of auto - hidrolysis are used with 'Belobrzesky' hemp variety that was grown in in Latvia. Hemp wood parts (shive) were obtained from the company "ZALERS" in Kraslava district, Latvia. In the shive fraction there still remains an average of $4-12 \%$ fibres [Andzs, 2010].

The SE samples depending on it pretreatment time could be defind as:

RUKK - industrial hemp dressing residues before treatment by steam explosion.

RUKK SE0.0 - industrial hemp dressing residues exploded immediately upon reaching the pressure of 3.2 Mp. 
RUKK SE0.5 - industrial hemp dressing residues after SE treatment for $0.5 \mathrm{~min}$.

RUKK SE1.0 - industrial hemp dressing residues after SE treatment for $1.0 \mathrm{~min}$.

ROKK SE3.0 - industrial hemp dressing residues after SE treatment for $3.0 \mathrm{~min}$.

Steam explosion (SE) is principally a rather simple process. The SE process shown schematically in Fig.2. The biomass is treated by saturated steam, usually at pressures up to 40 bars. The treatment time varies from some seconds to some minutes. After the treatment, within a split second, the biomass is decompressed (exploded) to the pressure of ambient atmosphere. Empirically, conditions of the steam explosion can be characterized by a single parameter comprising temperature and time - the severity parameter.

Empirically result of SEA is determined by severity parameter Ro expressed as:

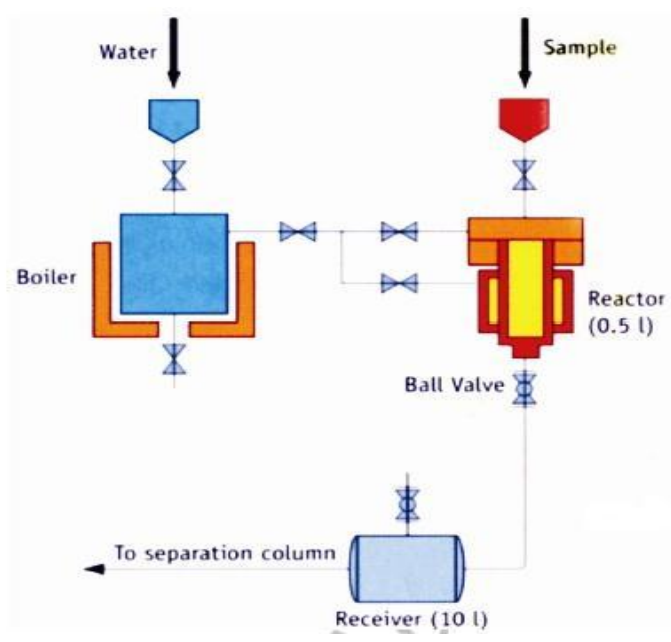

Fig. 2. Steam explosion unit [Gravitis et al. 2010].

$$
\log \operatorname{Ro}=t * \exp [(\mathrm{T}-100) / 14.75]
$$

Where duration of exposure to high pressure ( $t$, minutes) and temperature $\left(T,{ }^{0} \mathrm{C}\right)$ express the SE severity against the base temperature Tbase $=100{ }^{\circ} \mathrm{C}$. Similar SE results may be achieved with different combinations of $t$ and T. However, there is a contribution from other factors such as moisture content of the sample, size of particles etc [Gravitis et al., 2008].

Hemp shives of the 'Belobrzesky' hemp variety containing $6-11 \%$ moisture after drying at room temperature were used for experimental treatment by steam explosion. Parameters used in the steam explosion treatment were selected on the basis of previous research experience of the Latvian State Institute of Wood chemistry, Laboratory of Eco-Efficient Conversion of Biomass (Table 1).
TABLE1

SE PARAMETERS

\begin{tabular}{|c|c|c|c|c|c|}
\hline Parameter & $\begin{array}{l}\text { Time, } \\
\text { min. }\end{array}$ & $\begin{array}{l}\mathrm{T}, \\
0 \mathrm{C}\end{array}$ & $\begin{array}{l}\text { Pressure, } \\
\text { Mp. }\end{array}$ & $\begin{array}{l}\log \\
\text { R0 }\end{array}$ & $\begin{array}{l}\text { Loss } \\
\text { after } \\
\text { SE, } \\
\%\end{array}$ \\
\hline $\begin{array}{l}\text { Reference } \\
\text { Hemp } \\
\text { shives }\end{array}$ & - & - & - & - & - \\
\hline RUKK- 00 & 00 & \multirow{4}{*}{235} & 3.2 & 2.97 & 2.43 \\
\hline RUKK- 0.5 & 0.5 & & 3.2 & 3.67 & 6.27 \\
\hline RUKK- 1.0 & 1.0 & & 3.2 & 3.98 & 13.05 \\
\hline RUKK-3.0 & 3.0 & & 3.2 & 4.45 & 19.99 \\
\hline
\end{tabular}

Making the simple component fractionation of the pretreated material (all processes at room temperature), first, the soluble parts removed by adding water (1:4). Lignin is extracted from the residual by solving it in $0.4 \%$ solution of $\mathrm{NaOH}(1: 4)$ wherefrom it is precipitated by adding hydrochloric acid to neutralize the solution. The precipitated ligneous mass is rinsed in water to remove the remnant of sodium chloride before filtration. After drying in air the filtrate turns into powder presented as steam-exploded lignin [Gravitis et al., 2010].

\section{RESULTS AND DISCUSSION}

As seen from Figs. 3 and 4, the SE essentially affects the bulk density of hemp shives. The shorter processing time, the higher bulk volume of the processed substance. At small $\log \mathrm{R}_{0}$ the amount of extracted lignin is small.

(1)

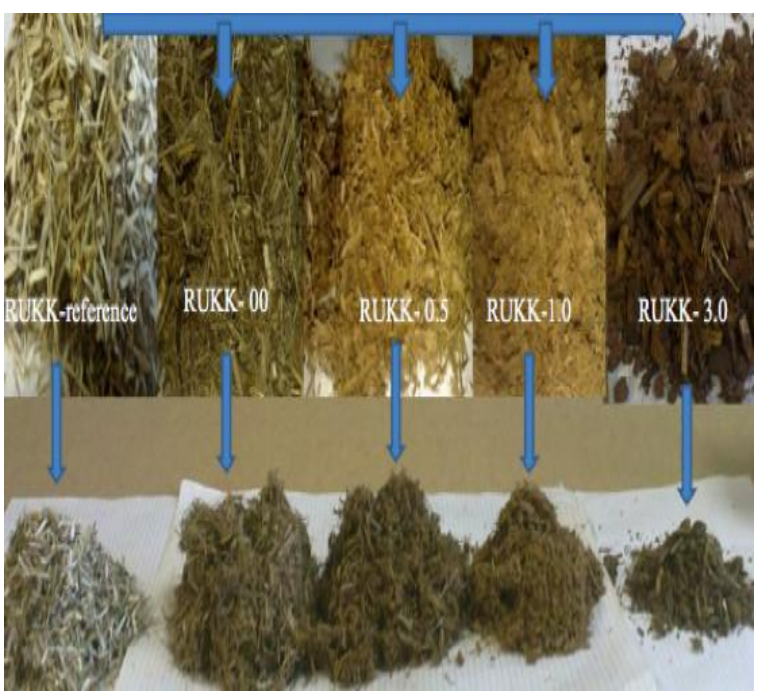

Fig. 3. Hemp shives after SE. 


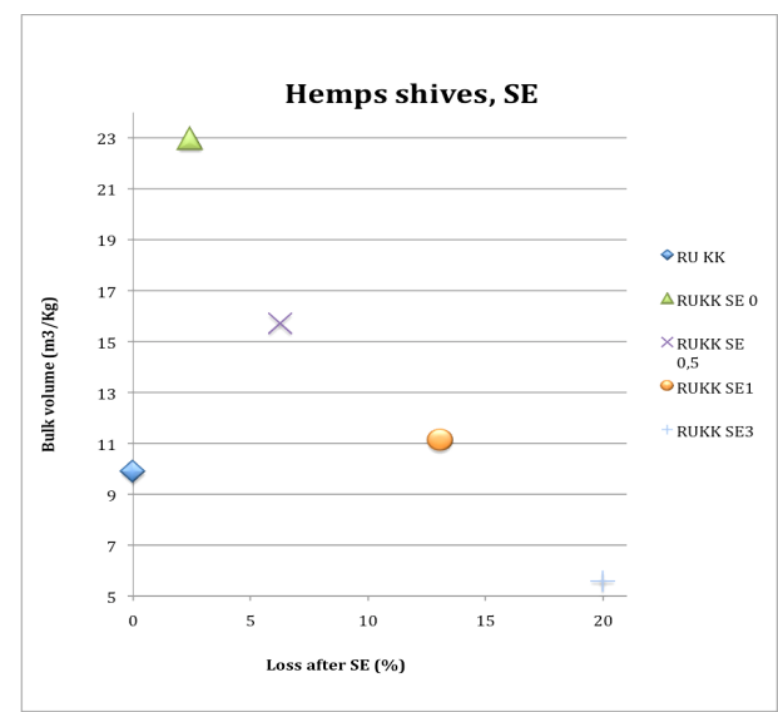

Fig. 4. Volume of loose steam exploded hemp dressing residues with relation to loss of massduring the treatment.

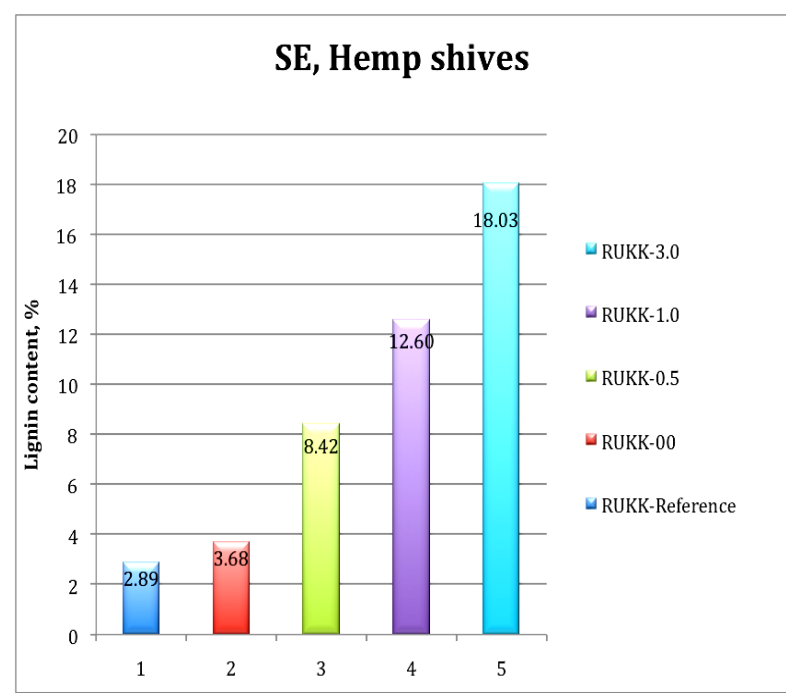

Fig. 5. Lignons extracted by alkali-solutions.

The first partial loss of the mass of hemp shives as indicated by weight loss and results of extraction after SE is due volatile sugars.
The amount of residual lignin in sample RUKK-3.0 treated at $\log \mathrm{R}_{0}=4.45$, points to complete collapse of the shives. The loss of mass is shown in Fig. 4, the extracted mass containing lignin - in Fig. 5. The SE results are affected by the moisture content of the material [Kaleine et al., 1990].

\section{CONCLUSIONS}

The resulting amount of Lignin is directly proportional to the $\mathrm{SE}$ conditions $\log \mathrm{R}_{0}$ the most important factor is the processing time. Mass loss and the resulting amount of lignin by steam explosion treatment is proportional to the severity factor. Among the samples RUKK-Reference and RUKK-00 a high bulk volume and should preferably be used as heat insulation material (Fig. 4).

\section{$\mathrm{V}$ ACKNOWLEDGMENTS}

Latvian State Institute of Wood chemistry, Laboratory of Eco-effective Conversion.

\section{REFERENCES}

[1] Andzs, M., Riga Technical university, Masters degree work (2010).

[2] Gargulak, J.D., Lebo, S.E., 2000. Commercial use of lignin-based materials. In: Glasser, W.G., Northey, R.A., Schultz, T.P. (eds.) Lignin: Historical, Biological, and Materials Perspectives. ACS Symposium Series. American Chemical Society, p. 307

[3] Gravitis J.1996. Material Separation Technologies and Zero Emissions. Proc. Second Annual UNU World Congress on Zero Emissions, Chattanooga, Tennessee, May 29-31, 168-173.

[4] Gravitis, J., Abolins,J., Kokorevics, A. 2008 Integration of biorefinery clusterstowards zero emissions, Environmental Engineering and Management Journal, 7(5):569-577.

[5] Gravitis, J., Abolins, J., Tupciauskas, R., Veveris, A. 2010. Lignin from Steam-exploded Wood as Binder in Wood Composites. Journal of Environmental Engineering and Landscape Management, Technika, Vilnius, 18(2): 75-84.

[6] Kaleine, A., Veveris, A., Polmanis. G., Erins, P., Belickis. J., Jakobsons, K., Kuzmane, V., Polmanis. G., Kaleine. A., Erins. P., 1990. Journal Wood chemistry, Latvia, Riga, N.3.100. (In Russian).

[7] Liepins. G., 1990. Lignin. Journal Wood chemistry, Latvia, Riga, N.3.106. (In Russian).

[8] Zakis, G., Basic of wood chemistry, SIA „Tipografija Perse”, [in Latvian], Riga, 2008, -p.34. 